\title{
Colour and inclusivity: a visual communication design project with older people
}

\author{
Fernando Moreira da Silva \\ CIAUD - Research Centre in Architecture, Urban Planning and Design, Faculty of Architecture, Technical \\ University of Lisbon. E-mail: fms.fautl@gmail.com
}

\begin{abstract}
In an ideal world, inclusive products and services would be the standard and not the exception. This paper presents a systematic approach to an overlap between Visual Communication Design, Printed Colour and Inclusive Design, for older people, with the aim to develop of a set of research-based ageing and ergonomics-centred communication design guidelines and recommendations for printed material (analogical displays). The approach included an initial extensive literature review in the area of colour, older people and ergonomics issues and vision common diseases, communication design. The second phase was the implementation of an experiment to measure the different colour experiences of the participants in two sample groups (one in UK and another one in Portugal), using printed material, to find out the colours one should use in analogical communication material, being aware of the colour contrast importance (foreground versus background) and the difficulties experienced by older people to read and understand lettering, signs. As main contribution of this research project, we developed a set of guidelines and recommendations based on the reviewed literature and the sample groups' findings, trying to demonstrate the importance of these guidelines when conceiving a new communicational design project in a way this project will achieve vision comfort and understandability, especially for older people, in an inclusive design perspective.
\end{abstract}

Keywords: Inclusive Design, Colour, Older People, Ergonomics, Understandability

\section{Introduction}

This paper summarises the content and output of a 18 months scoping research study concerned with colour and visual communication in analogical support for older people. The output from this project is the initial stage of colour guidance and recommendations for older people, community care professionals and designers. Throughout the project the research team consulted an advisory panel whose members represented a wide range of disciplines, among which colourists, communicational designers and older people associations.

To work in Visual Communication one needs to have knowledge of different techniques and how to manipulate them. However, there is a gap in knowledge of Colour and Inclusive Design. Most of the studies that have been implemented until now focus on the use of colour and text in digital displays, not in analogical supports.
The research object of this study is the overlap between Visual Communication Design, Printed Colour and Inclusivity, for older people.

The main objective is to introduce colour as a variable of great importance in Visual Communication, in an Inclusive Design perspective, having as a target older people.

Other objectives of this research project are:

- to measure the different colour experiences of the participants (having two sample groups:

- one in UK and another one in Portugal);

- to find out the colours one should use in printed communication material, being aware of the colour contrast importance (object versus background) and the difficulties experienced by older people, of understanding the communicated object (lettering, signs), using printed material;

- to identify important key points and draw recommendations for designers' policy when using colour, in a way of improving visual communica- 
tion design quality, infusing an inclusive design perspective.

An inevitable outcome of the ageing population is an increase in the difficulty of reading and understanding visual printed messages (analogical support) due to the lack of information about colour, mainly for communicational designers, in an inclusive design perspective. Until now, little research has been carried out on how colours in form and background relationship affect older people. If text does not have sufficient contrast compared to its background, people will have problems.

This study is aimed at addressing this lack of evidence-based data. As well as a literature search, the project has involved two sample groups of older people between 65 and 87, in Marple (Great Manchester, UK) and in Cascais (Lisbon District, Portugal).

The purpose of the sample groups was to test and validate information gathered during the literature review and to generate other findings for future development and well as to identify research needs.

A range of results has been obtained from this study and these results are summarised in this work.

\section{Context}

In an ideal world, inclusive products and services would be standard and not the exception.

Collaboration between users, designers and producers from the very beginning of the project until its completion, would be the key for success. However, in the real world it isn't like this. Designers complain about the lack of tools and information necessary to deliver inclusive products and services for their clients. What is available, they say, is in a language that they can't understand and in shapes difficult to implement. The producers point out constrictions of time and cost, besides the lack of appropriate information at enterprise management level for inclusive design. They can be aware of the demographic reality in what concerns older people all around the world, about the pension crises and the potential market, but they have difficulties in visualising precisely in what way inclusivity can be applied to their actual or future range of products or services, or even its relevance for the product innovation. Meanwhile, "critical" users who could supply the creative stimulus that designers need are still out of the process.

To work in the Graphic Design and Visual Communication area one needs to have knowledge of different techniques and how to manipulate them. Despite the knowledge in this professional circle, there is a gap in knowledge of Colour and Inclusive Design. The interpretation of visual information, as well as semiotics is closer to meaning interpretation, is consequently highly subjective and projective. Gibson [8] affirms that visual perception is "richer and more open to personal interpretation".

The visual and non-verbal systems operate relatively without a "tutor" in our society, at least in comparison with language. Visual communication is a primary system, which is located at the same expression level as is verbal language. In terms of human development, the visual system of signs occurs earlier than language itself. In terms of complexity, one can find visual interpretation more complex than verbal interpretation, because of the absence of a conventional sign system and the formalization of a training protocol. Visual communication could also be considered primary because the observer has to learn how to control better, and independently, the visual interpretative function.

Finally, visual communication is neither derivative nor peripheral to verbal language, and consequently the designation of visual communication as secondary, tertiary or of a "superstructure" built in verbal language is not right. In Paivio notion [23] [24], a more appropriated model would be built of a dual codification, saying that visual and verbal information are codified and uncodified by perceptive and cognitive separated systems. A system is considered visual/pictorial and manipulates simultaneously the elements of imagination; the other one is linguistic and propositional and operates in sequence.

Messaris [20] explains that the two systems are structural and functionally distinct. Although being independent, the two systems are also interdependent in a way that a certain visual concept may be converted in a verbal etiquette and vice versa. A new interpretation to explain the interaction between the two systems is the metaphor of processing interactively in parallel. Redefining the notion of a "primary" system, and including visual communication as well as the verbal one, we can achieve a more complete analysis of the complexity of communication.

\section{Detailed description}

The Project overlaps different areas and knowledge, among which:

- Colour contrasts and colour measurement;

- Light sources (natural and artificial); 
- The evaluation of the proprieties of the light sources and the influence of the surfaces;

- The evaluation of the colour aspects inside visual communication area;

- Legibility and the obstacles to reading;

- Older people and visual limitations.

To accomplish this, a literature review considered relevant for the study was undertaken.

The work with experts in the area of Colour, Visual Communication, Inclusive Design and Older People, as well as with the users and the associations of people with impairments, was also fundamental for the study developed. Also the direct field work with the users (sample groups), was a factor of great importance for the present study.

A qualitative method was chosen in order to allow a detailed exploration and the handling of complex and diverse information. A structured questionnaire was designed and used with the two sample groups of older people ( $65-87$ years). The obtained data was used to identify and describe the issues raised and to consider replication, linkages and differences.

This study doesn't intend to provide numerical data. To measure the different colour experiences of the participants, we used only printed material.

\section{Older people and visual impairment}

As the population is growing and ageing, the proportion of older people is expected to increase. One of the effects that growing older has on vision is that, on average, less light falls on the retina, and there is less tolerance to glare. Statistics from the Department of Health in UK [4] clearly show that a large percentage of people both blind and partially sighted people are 75 and over. Information from the RNIB [25] suggests that the most common eye pathologies are: macular degeneration, cataract, glaucoma, retinitis pigmentosa and diabetic retinopathy. Loss in the fovea affects visual acuity and colour perception and general loss of vision across the whole visual field. Friström and Lundh [11] arrived to the conclusion that peripheral colour contrast sensitivity was affected by cataract, even when only moderately developed. This finding is of importance and should be considered when the method is used to study other eye diseases e.g. glaucoma. Central colour contrast sensitivity was also affected by cataract.

Maintenance and Refurbishment Programme used these resulting effects to propose three categories: central, peripheral and general visual field loss [2].
Macular degeneration, one of the most common causes of visual impairment, makes close up tasks such as reading more difficult. This can, in some cases, be helped by the use of optical aids.

Impairment of peripheral vision can result in 'tunnel vision', which can make it difficult to spot potential hazards. Retinitis pigmentosa, macular degeneration and cataracts can result in problems or discomfort with glare and bright lights or backgrounds.

Vision is one of the primary senses and serious or complete loss of sight also has a major impact on a person's ability to communicate effectively and function independently [13]. Lakowski and Drance [16] found that a large number of patients with ocular hypertension $(\mathrm{OH})$ showed acquired colour vision losses. These losses were particularly in the bluegreen part of the spectrum, the called tritan defects [14]. They seemed to precede nerve fibre bundle defects in the visual field [5]. The loss of chromatic sensitivity in the short wavelength part of the spectrum in glaucoma was confirmed by others. Short wavelength automated perimetry (SWAP) has been shown to detect glaucoma damage earlier than conventional white-on-white perimetry and also to be more sensitive to progression of visual field loss, and to progression of glaucomatous disc cupping [10]. Other eye diseases, such as low-grade type 2 diabetic retinopathy [9] and moderate cataract [11] have been shown to affect peripheral colour contrast. Central colour contrast sensitivity is affected by macular degeneration, even with early age-related maculopathy [8]. In the UK, loss of sight most frequently happens later in life and as part of the ageing process. Recent figures [6] suggest that more than $12 \%$ of people over 75 have some sight loss. Visual acuity is reduced by $10 \%$ for $60-69$ year olds, $30 \%$ for $70-79$ year olds and $35 \%$ in the over- 80 s. In the UK, 65,000 people are diagnosed with low vision each year [21].

With age, changes to the eye increase sensitivity to glare, difficulty of adapting to changing light levels, and make contrast and colour harder to discern. A good colour use for visual communication, as well as helping to improve visual performance, it may also increase general well-being and health. The effect of sight ageing is partially attributed to the yellowing of the retina, lens and vitreous humor yellow with age causing colours such as violet, blue and green to be filtered out, reducing the contrast sensitivity of the eye [14] and increasing the requirement for light in older subjects [3]. The effect of age on measurement legibility is further compounded by a reduced ability of the iris to dilate, under all light conditions. 


\section{Human striate cortex and colour contrast processing}

According to Weiskrantz et al [27], colour appearance is not simply determined by the composition of reflected light. Colour appearance is more closely related to a constant property of a surface, its spectral reflectance. The relative reflectances of adjacent surfaces at any specific wavelength of light determine the relative intensities of light reflected from them at that wavelength, regardless of the spectral composition of the light illuminating them. Ratios of cone signals are approximately invariant under changes of illumination [7]. If the reflectance properties of one surface are known, then the properties of all other surfaces seen under the same illuminant can be inferred on the basis of these cone ratios or colour contrasts (this is the essence of Land's "retinex" colour constancy algorithm) [17].

Questions about the role striate cortex, the first visual area of the higher mammalian brain, plays in colour perception have been reignited recently [13]. These cells in striate cortex are excited by light of one distribution of wavelengths and inhibited by light of another distribution falling in one small region of space. Adjacent or surrounding regions show the opposite pattern of responses. The cells therefore respond optimally to borders with a specific chromatic contrast that fall across the centre and surround regions of their spatial receptive fields. These findings suggest colour-specific processing starts earlier in the visual system than previously thought. The earliest mechanisms of colour constancy, our ability to recognize objects' colours regardless of the colour of light illuminating them, might occur in striate cortex. If so, people without striate cortex, like some older people, will lose colour contrast processing. They should behave as if objects change colour as the light illuminating them changes, their apparent colour depending on the wavelengths of light they reflect, rather than the properties of their surface material [27]. The influence of contrast in reading is important not only because text of a wide range of contrasts is encountered in the environment but also because many ocular conditions lower the effective contrast of the reading stimulus. Most studies of the role of contrast in reading, however, have treated only the luminance dimension. In general, reading is found to be fastest when the luminance difference between text and background is maximal. For example, using high-contrast edges, Legge and Rubin [18] demonstrated that for observers with normal vision the lu- minance contrast and the background luminance (or the text luminance when the background was dark) determine reading rate regardless of the colour of the text [15]. Tinker and Paterson [26] found the legibility of coloured inks on differently coloured papers to depend primarily on the luminance difference between the text and the background, but the range of conditions that they could examine was limited by the nature of their stimulus medium. Lippert [19] reported that legibility of briefly presented digits depended on the colour difference between the digits and the background. While Lippert systematically varied luminance and chromatic differences between text and background, he used a range of character sizes that was near the acuity limit of the chromaticcontrast-sensitivity function. Many eye conditions that result in low vision also result in colour vision deficiencies. While the most common congenital colour defects produce little in the way of performance limitations in and of themselves, the additional loss of sensory information that arises from a colour defect for an already compromised visual system could have greater consequences. Conversely, the residual colour discrimination that some people with low vision show could provide a viable avenue along which information could be presented to enhance performance on certain tasks dependent upon visual input. This line of reasoning suggested that a useful approach would be: a) to evaluate what residual colour discrimination low vision people display and b) to evaluate what influence the introduction of chromatic contrast might have on specific tasks for normal and low vision people [1]. With knowledge of the colour contrasts that an individual discriminates best, the degree to which colour cues influence performance on specific tasks can be evaluated.

A major goal of our colour contrast work is the dissemination of simple, comprehensible guidelines for choosing colours that work effectively for all, including those with the acquired colour deficiencies that almost always accompany low vision, and those with congenital colour deficiencies.

\section{Methodology}

After a literature review of the relevant material we were able to produce the State of the Art, from which emerged this hypothesis for our study:

To produce a more inclusive design project when designing visual communication analogical products, designers must be aware of the issues related with 
colour and text legibility, due to the reading problems experienced by many users, like older people.

For the second stage of this research project, we decided to develop an experiment (active research) using two sample groups of older people with the same gender composition and general characteristics. The process involved development of tools to work with the groups, especially printed colour material always relating front and background colours. The search was carried out in several occasions (rounds), using the same material in similar lighting conditions and the same distances. During the experiment sessions, the sample groups of older people who took part in this study had the light up, on average, 900 lux (illuminance), as recommended by O'Neill [22] in a study conducted by a Research Group for Inclusive Environments at the University of Reading, in terms of having a "Good" colour rendering. The examples were very simple and designed to be readily understood. They were written in plain English for the UK sample group and in Portuguese for the Portuguese group. For this reason no technical terms were required when participants were asked to read the different words and sentences. The words and sentences acted as forms, using different colour schemes, in form/background relationship. Participants in the sample groups were volunteers recruited by the research team, among their acquaintances. In total, 8 people volunteered to take part in the UK sample group: 5 female persons and three men, with ages comprised between 65 and 85 , all in sight normal conditions for people in this range of age, only with aged vision, but with no specific sight diseases.

In the Portuguese sample group 8 people took part in it: also 5 female persons and three men, with ages comprised between 65 and 87, all with the same sight characteristics as the UK group. Several messages, in different colour contrasts (see example in Figure 1) were printed on A4 format cards for a total of 24 .

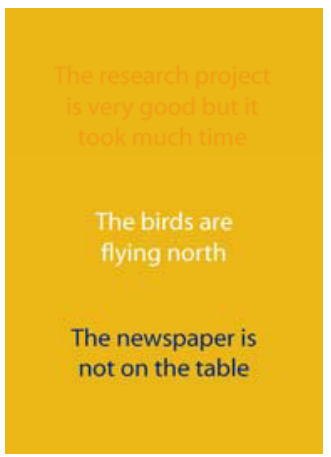

Fig1. - Example of card used during the experiment
All messages were created using 48 point Myriad. This size was chosen, according to the following formula:

$$
\text { Lettering high }(\mathrm{mm})=\underline{\text { Eye distance }(\mathrm{mm})}
$$

This is the reference formula for graphic designers, in terms of text legibility. The lettering size was chosen having in mind that the group members would be placed at a two meters distance from the cards.

For the colour production, we used the Pantone Matching System (PMS) Colours. The colours used in this research are colours that the researcher found more appropriated for the research aim and the target group. For this experiment, the inside level of light was measured by an A.W. Sperry light meter. Two sample groups of subjects (with ages 65-87) were tested to measure the legibility of 24 cards. Information for each group included the gender, age, requirement for eye wear. The results for colour blindness were recorded as "normal" for all group members. To be selected for each of the sample groups, the members could have any eye disease, only older vision.

After informed consent had been obtained, and the subjects' visual acuity and ability to perceive colour had been tested, each one was seated in front of a researcher holding the A4 cards, with a 2 meters distance between them, and asked to look to each card (see fig 2).

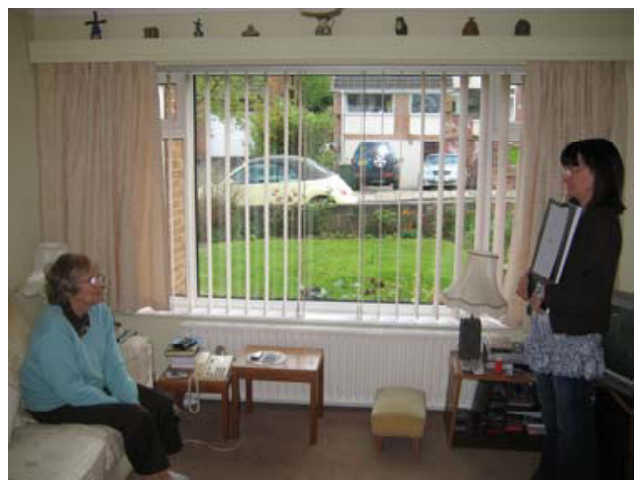

Fig. 2 - Experiment session with one of the members of the target group in UK

They were told to read the text written in each card, where object (text) and background had different colour combinations. There were also cards with different types of lettering and spacing, but using always the same letter dimensions. We also wanted to test the level of legibility and the eventual experi- 
enced difficulty, as well as the level of eye comfort and colour contrast. After the implementation of the experiment with the two sample groups of older people, we could achieve findings, which were confronted with the drawn hypothesis. We were able to verify that not only we had proved the hypothesis but also we had amplified the initial knowledge with a contribution for the study area.

\section{Research findings}

With the sample groups, the research team verified some conclusions from the literature review and found some solutions for an inclusive approach in visual communication design, using colour in analogical material, for older people. The different rounds of tests allowed the research team to identify the main problems with colour use for visual communication for older people. During the experiment developed with both sample groups there were immediately negative comments about some colour combinations. Examples are: "Have trouble with reds," "Reds are hard," "Black on red is terrible," "Yellow on red is not a good combination," "I hate reading on red paper," "Yellow on red is very hard to read".

After the experiment with the UK sample group we have achieved the following results:

- All the participants had a good legibility when the contrast between background and object (word or sentence) was clear, such is the case of the background in white, light grey or yellow and the object in dark blue or black; or the background in dark grey, dark blue, red or black, and the object in white or yellow;

- Three of the participants had experienced difficulties with the orange over the yellow;

- The green on pink was really difficult for 6 of the participants;

- None could read black on dark grey, or yellow on white;

- Five of the participants had difficulties of legibility with green on red, or light blue on red;

- White on light grey was very difficult to read for 6 participants;

- Five participants experienced difficulties with light blue on black;

- Three had difficulties with light grey on light blue, or red on dark blue;

- Two had difficulties when reading red on light blue;
- Four participants had problems when reading purple over light blue;

- None could read orange on dark yellow. The experiment also included other aspects besides colour contrast, concerning the type of lettering; the use of uppercase letters and upper and lower case letters; the space between letters; the line spacing; and the type of paper, with glare and non-glare coverings.

When working with the Portuguese sample group, we have achieved the following results:

- All the participants had a good legibility when the contrast between background and object was clear, such is the case of the background in white, light grey or yellow and the object in dark blue or black; or white or yellow on dark grey, dark blue, red or black;

- Four of the participants had experienced difficulties with the orange over the yellow;

- The green on pink was really difficult for 7 of the participants;

- None could read black on dark grey, or yellow on white;

- Five of the participants had difficulties of legibility with green on red, or light blue on red;

- White on light grey was very difficult to read for 5 participants;

- Four participants experienced difficulties with light blue on black;

- Four had difficulties with light grey on light blue;

- There were no difficulties when reading red on light blue;

- Six participants had problems when reading purple over light blue;

- None could read orange on dark yellow;

- Three had difficulties when reading red on dark blue.

Comparing the results of the two sample groups:

- All the participants had a good legibility when the contrast between background and object (word or sentence) was clear, such is the case of the background in white, light grey or yellow and the object in dark blue or black; or the background in dark grey, dark blue, red or black, and the object in white or yellow;

- Seven in sixteen of the participants had experienced difficulties with the orange on yellow;

- The green on pink was really difficult for 13 out of 16 of the participants;

- None could read black on dark grey, or yellow on white; 
- Ten in sixteen had difficulties of legibility with green on red, or light blue on red;

- White on light grey was very difficult to read for 11/16 participants;

- Nine in sixteen participants experienced difficulties with light blue on black;

- Seven in sixteen had difficulties with light grey on light blue;

- Ten in sixteen participants had problems when reading purple over light blue;

- None could read orange on dark yellow;

- Six in sixteen had difficulties when reading red on dark blue.

In what concerns the other aspects of the reading problems, all the participants in both groups $(100 \%)$ had the same opinion, as follow:

- Type of lettering: they all had difficulties with complicated, decorative or cursive fonts;

- Standard serif or sans-serif fonts, with familiar, easily recognizable characters are best;

- The use of uppercase letters and upper and lower case letters: They were much more comfortable with the use of upper and lower case letters;

- The space between letters: they couldn't read the words or sentences which had no space between letters;

- Line spacing: they all experienced difficulties if there were no line spacing;

- Leading, or spacing between lines of text, should be at least 25 to 30 percent of the point size;

- Type of paper: much more difficult to read if the paper has a glare covering (reflecting surface).

In general terms, the only important difference between the two tables is that the Portuguese sample group had no difficulties when reading red on light blue whilst some of the participants in the UK sample group had some reading difficulties with this contrast

\section{Conclusion}

After the literature review implemented during the research and the experiment with the two sample groups, we achieved the following conclusions:

- After further analysis, the contrast combinations were separated into two groups: most legible and least legible;

- There is a good legibility when the contrast between background and object (word or sentence) is clear, such as:

○ the background in white, light grey or yellow and the object in dark blue or black;
○ the background in dark grey, dark blue, red or black, and the object in white or yellow;

- Green on pink or white on light grey are really difficult to read;

- Black on dark grey, orange on dark yellow or yellow on white are not effective;

- Green on red, red on dark blue, red on light blue or light blue on red are difficult to read;

- There are legibility difficulties with light blue on black, purple over light blue or orange on yellow;

- Light grey on light blue is not effective;

- Decorative or cursive fonts are difficult to read;

- Standard serif or sans-serif fonts, are best for the legibility;

- The use of uppercase letters is not very comfortable;

- The use of upper and lower case letters is very comfortable;

- The words or sentences which had no space between letters aren't effective;

- There are difficulties if there is no line spacing;

- Leading, or spacing between lines of text, less than 25 to 30 percent of the point size are not effective;

- Paper with a glare covering (reflecting surface) is not good for the legibility.

\section{Design Guidelines}

This study represents a scoping project to investigate colour and visual communication problems for older people, using analogical supports, and it provides useful context and background information, which confirms that there has been relatively little work on colour and analogical communication design and even less focusing on the needs of older people that naturally have aged vision.

After the research project conclusions, it is possible to design some guidelines for an inclusive visual communication project for graphic printed material:

- An objective measurement of legibility is a useful tool for the designer who wants assurance that his design will be easy to read;

- When one creates visuals that are intended to be read, it is important to have enough contrast between the background and the text;

- The human eye requires contrasts for visibility and legibility;

- The more an object contrasts with its surrounds, the more visible it becomes; 
- When the colour value is too close between text and background colours, it can create legibility problems;

- Too much contrast or the use of complementary colours take the idea of contrast too far: colours will appear to "vibrate" and will create legibility problems;

- Colours that are close in value tend to blur together, and their borders "melt.";

- Black on red should be avoided when designing printed material;

- Black text on a dark blue background or black type on a red background are hard to read;

- On a blue background yellow jumps out at the reader;

- Yellow text on a red background is difficult to read, possibly because of the poor contrast provided by this combination;

- Black text on a white background is the most legible contrast combination for all age groups;

- Patterned backgrounds or an image in the background of text reduces its legibility;

- The most common forms of colour blindness are associated with the inability to discriminate red and green wavelengths, so it's not effective to use red on green;

- Bright colours can produce glare, which might distract the user and cause the eyes to become tired;

- Paper with a reflecting surface is not good for the legibility.

\section{References}

[1] Arditi, A. \& Knoblauch, K. 1996. "Effective color contrast and low vision" in B. Rosenthal and R. Cole (Eds.) "Functional Assessment of Low Vision". Mosby. St. Louis. pp 129 135.

[2] Bix, L. 1998. "The effect of subject age on legibility". Unpublished Master's Thesis, Michigan State University, East Lansing.

[3] Bright, K, Cook, G, Harris, J. 1997. "Colour, Contrast \& Perception - Design Guidance for Internal Built Environments". The University of Reading.

[4] Department of Health. 2000. "Registered Blind and Partially Sighted People Year Ending 31March 2000”. Rep. A/F 2000/7, Department of Health, London.

[5] Drance, S.M., Lakowski, R., Schulzer, M. \& Douglas G.R. 1981. "Acquired colour vision changes in glaucoma". Arch Ophthalmol 99. pp 829-831.

[6] Evans, J.R., Fletcher, A.E., Wormald, R.P.L. et al 2002 "Prevalence of visual impairment in people aged 75 years and older in Britain: results of the MRC Trial of Assessment and Management of Older People in the Community". British Journal of Ophthalmology, 86. pp 795-800.

[7] Foster, D.H. \& Nascimento, S.M.C. 1994. Proc R Soc London Ser B 257. pp 115-121.

[8] Frennesson, C., Nilsson, U.L. \& Nilsson, S.E.G. 1995. "Colour contrast sensitivity in patients with soft drusen, an early stage of ARM". Doc Ophthalmol 90. Blackwell Publishing. pp 377-386.

[9] Friström, B. 1998. "Peripheral and central colour contrast sensitivity in diabetes". Acta Ophthalmol Scand 76. Blackwell Publishing. pp 541-545.

[10]Friström, B. 2002. "Colour contrast sensitivity in ocular hypertension. A five-year prospective study". Acta Ophthalmologica Scandinavica, 80(2) April. Blackwell Publishing, pp. 155-162.

[11]Friström, B. \& Lundh, B.L. 2000. "Colour contrast sensitivity in cataract and pseudophakia". Acta Ophthalmologica Scandinavica. 78(5). Blackwell Publishing. pp. 506-511.

[12] Gibson, J. J. 1971. "The Information Available in Pictures". Leonardo. 4 (Winter), pp. 27-35. Johnson, E.N., Hawken, M.J. \& Shapley, R. 2001. "Nat Neurosci”. 4, pp. 409-416.

[13]Jones, R. \& Trigg, R. 2007. "Dementia and serious sight loss". Bath: Research Institute for the Care of the Elderly, St Martin's Hospital.

[14]Kelly, M. 1993. "Visual impairment in the elderly and its impact on their daily lives". Unpublished PhD Dissertation, Texas Woman's University.

[15] Knoblauch, K. et al 1991. "Effects of chromatic and luminance contrast on reading”. J. Opt. Soc. Am. A. 8(2), p 428.

[16]Lakowski, R., Drance, S.M. 1979. "Acquired dyschromatopsias: the earliest functional losses in glaucoma". Doc Ophthalmol Proc Ser. 1979, 19, p159.

[17]Land, E.H. \& McCann, J.J. 1971. J Opt Soc Am, 61, pp1-11.

[18] Legge, G.E., Pelli, D.G., Rubin, G.S. \& Schleske, M.M 1985. "Psychophysics of reading". I. Normal vision. Vision Research, 25, pp239-252.

[19] Lippert, T.M. 1986. "Color difference prediction of legibility performance for CRT raster imagery", in "Digest of the Society for Information Display" (Society for Information Display, Playa Del Rey, California, 17, pp. 86-89.

[20] Messaris, P. 1994. "Visual Literacy: Image, Mind, \& Reality". Boulder CO: Westview Press

[21] Morris, C. 1999. "Visual impairments and problems with perception". Journal of Dementia and Care, Nov/Dec, pp2628

[22] O'Neill, L. 2003. "Lighting the homes of people with sight loss". The University of Reading.

[23] Pavio, A. 1971. "Imagery and Verbal Processes". NY: Holt, Rinehart and Winston.

[24] Pavio, A. 1986. "Mental Representations: A Dual Coding Approach". Oxford: Oxford University Press.

[25] RNIB, Health Promotion England 2001. Fact sheet 3 "Older people, visual impairment and accidents", London.

[26] Tinker, M.A. \& Paterson, D.G. 1931. "Studies of typographical factors influencing speed of reading". VII. "Variations in color of print and background". Journal of Applied Psychology 15, pp. 471-479.

[27] Weiskrantz, L. et al 2007. "Color contrast processing in human striate cortex". USA: PNAS. 